Annuaire suisse de politique de développement

26-2 | 2007

Financer le développement par la mobilisation des ressources locales

\title{
Le financement du développement par l'aide budgétaire générale : premier bilan et perspectives à moyen terme
}

Jean-Luc Bernasconi

\section{CpenEdition}

\section{Journals}

Édition électronique

URL : http://journals.openedition.org/aspd/146

DOI : 10.4000/aspd. 146

ISSN : 1663-9669

Éditeur

Institut de hautes études internationales et du développement

Édition imprimée

Date de publication : 1 novembre 2007

Pagination : 197-213

ISBN : 978-2-88247-068-3

ISSN : 1660-5934

\section{Référence électronique}

Jean-Luc Bernasconi, « Le financement du développement par l'aide budgétaire générale : premier bilan et perspectives à moyen terme ", Annuaire suisse de politique de développement [En ligne], 26-2 2007, mis en ligne le 22 juin 2009, consulté le 07 septembre 2020. URL : http://

journals.openedition.org/aspd/146; DOI : https://doi.org/10.4000/aspd.146 


\title{
Le financement du développement par l'aide budgétaire générale: premier bilan et perspectives à moyen terme
}

\author{
Jean-Luc Bernasconi*
}

'objectif de cet article est de présenter un état des lieux de l'instrument de l'aide budgétaire générale tel qu'il est appliqué par la Suisse depuis le début des années 2000 environ, notamment à la lumière d'évaluations récentes. Il en ressort que les résultats atteints par l'aide budgétaire sont globalement positifs, mais qu'un certain nombre d'opportunités et de défis doivent être relevés pour améliorer encore l'efficacité de l'instrument. Ainsi, nous concluons que l'application de cet instrument devrait être poursuivie même si l'approche sélective reste de mise pour un bailleur aux ressources limitées comme la Suisse. L'analyse des effets de l'aide budgétaire permet également d'apporter un éclairage sur l'approche de la Suisse à l'amélioration de la gestion des finances publiques, et d'aborder certaines questions transversales touchant l'aide budgétaire telles que la corruption, la décentralisation ou le renforcement de mécanismes de responsabilisation des Etats bénéficiaires et donateurs.

L'aide budgétaire générale a émergé à la fin des années 1990 comme un instrument novateur de la coopération financière au développement. Certains pays donateurs, telle la Suisse, ont assez rapidement adopté cet instrument, quand bien même de manière très ciblée, avec des objectifs quant aux effets d'échelle qu'il pourrait développer par rapport à la coopération fournie plus traditionnellement, par exemple sous forme de projets.

L'aide budgétaire générale se définit comme un transfert de ressources financières en faveur du trésor du pays partenaire, pour soutenir ce pays dans l'exécution de son budget national (dépenses de fonctionnement et d'investissement). L'aide budgétaire est dite "générale» quand elle n'est pas affectée à un secteur particulier. Les ressources sont gérées par les Etats partenaires selon les règles de gestion des finances publiques en vigueur dans le pays. Le décaissement des ressources est conditionné par un catalogue de réformes et d'indicateurs, dont la définition et l'examen régulier sont l'objet d'un dialogue politique entre bailleurs et pays partenaires. La performance est ainsi au centre de l'aide budgétaire générale. Comme l'élément «performance» introduit une incertitude quant au volume final d'aide mis à disposition, le séquencement des examens de performance est agencé en coordination étroite avec le calendrier de planification budgétaire des pays partenaires, répondant ainsi au besoin de prévisibilité des flux d'aide. C'est d'ailleurs parce que l'aide budgétaire est conforme à la plupart des principes arrêtés par le Comité d'aide au développement (CAD) de

* Chef de secteur, Soutien macroéconomique, Coopération et développement économiques, Secrétariat d'Etat à l'économie (SECO), Berne. 
l'Organisation de coopération et de développement économiques (OCDE) que la Déclaration de Paris a recommandé un recours croissant à l'aide budgétaire générale (voir ci-dessous). En résumé, l'aide budgétaire générale comprend un véritable paquet de mesures complémentaires au transfert des ressources financières, incluant le dialogue politique autour de la conditionnalité, l'harmonisation des pratiques des bailleurs, l'alignement sur des priorités définies par les pays partenaires et le renforcement des capacités par une meilleure coordination de l'assistance technique. La suite de cet article montre comment ces objectifs ambitieux se sont concrétisé après une demi-décennie de mise en œuvre.

\section{L'aide budgétaire: une évolution logique de l'appui macroéconomique}

Même si l'on présente souvent l'aide budgétaire générale comme un instrument novateur, son apparition, ou plutôt sa transformation dans la forme actuelle, est le résultat des enseignements tirés lors de l'application d'autre appuis financiers de nature macroéconomique. La Suisse aussi, et le Secrétariat d'Etat à l'économie (SECO) en particulier, a pu faire ce cheminement. L'aide budgétaire générale a succédé à l'aide à la balance des paiements, qui était fournie épisodiquement à des pays faisant face à des déséquilibres soudains et marqués de leurs comptes extérieurs, par exemple à la suite de chocs défavorables sur leurs termes de l'échange. L'objectif était alors centré sur la stabilisation macroéconomique à court terme, par un apport en devises permettant le financement d'importations pour permettre à l'économie de continuer à fonctionner. Dans certaines instances, ce financement était «lié», dans la mesure où l'on ne finançait que des importations en provenance du pays bailleur de fonds. Le contrôle et l'interaction entre pays bailleur et pays bénéficiaire pouvaient ainsi se limiter essentiellement à une vérification des factures d'importation à concurrence du montant d'aide octroyé, le dialogue politique étant essentiellement «délégué» aux programmes des institutions financières internationales (Fonds monétaire international et Banque mondiale). Même si l'objectif poursuivi, à savoir contribuer à éviter une crise de la balance des paiements, était atteint à court terme, ce type d'appui ne s'avérait guère soutenable dans la durée et n'offrait pas de réel soutien à la mise en œuvre d'une politique de développement social et économique à moyen terme.

Avec l'émergence des stratégies de réduction de la pauvreté et d'une réflexion plus globale sur les politiques de développement au cours des années 1990 est apparu le besoin de développer des instrument de nature programmatique, c'està-dire visant à une plus grande cohérence entre l'action décentralisée, voire parfois isolée, des projets et les appuis macroéconomiques. Avec l'avènement des Objectifs du Millénaire pour le développement (OMD), il est également apparu impératif de rechercher des résultats à grande échelle et de tenter de résoudre ce que l'on pourrait appeler un paradoxe «micro-macro», qui décrit la situation où les projets des bailleurs semblent obtenir individuellement des résultats probants alors même qu'il est impossible d'observer un impact positif de tous ces efforts à un niveau agrégé (celui des indicateurs nationaux par exemple). Les appuis programmatiques, telle l'aide budgétaire générale, ont précisément pour dessein de mettre en cohérence les objectifs des politiques macroéconomiques et des politiques sectorielles au travers du budget des Etats partenaires. Auparavant, 
l'alignement sur l'objectif macroéconomique prévalait (par exemple le solde budgétaire, avec comme cibles intermédiaires la masse salariale dans la fonction publique ou le plafonnement des dépenses de fonctionnement) et était déterminé de façon isolée dans les programmes d'ajustement, parfois sans prise en compte des objectifs sectoriels ni des allocations budgétaires correspondantes. En outre, l'appui macroéconomique ayant été souvent appliqué selon le principe de l'urgence des besoins, il n'était pas compatible avec une concentration géographique et s'avérait peu propice au développement de synergies avec d'autres instruments mis en œuvre par la Suisse. En d'autres termes, l'aide à la balance des paiements était généralement pratiquée de façon plutôt ad hoc, séparément du reste de l'effort de coopération.

Même si l'objectif de stabilité macroéconomique demeure aujourd'hui absolument central, l'aide budgétaire générale a marqué une rupture avec l'aide à la balance des paiements et avec le «tout macroéconomique», dans la mesure où elle s'inscrit dans une perspective plus longue et place un accent particulier sur la stratégie de réduction de la pauvreté et de promotion de la croissance des pays bénéficiaires. Par l'aide budgétaire, on cible des pays partenaires qui ont fait leurs premières preuves en matière de réformes économiques et sociales et démontré un engagement crédible pour poursuivre, voire accélérer ces réformes.

Même s'ils prennent souvent la forme de dons (exclusivement dans le cas de la Suisse), ces appuis ne sont pas pour autant des «chèques en blanc», puisque leur décaissement dépend de la réalisation d'un certain nombre de critères de performance communément agréés entre pays bailleurs et bénéficiaires et faisant l'objet d'un examen régulier. Le caractère conditionnel des déboursements n'est pas nouveau puisqu'il a aussi marqué les programmes d'ajustement structurel (PAS) des années 1980, si souvent décriés. L'aide budgétaire décrite ici est dite de «partenariat» (partnership general budget support) dans la mesure où elle tente de se distinguer précisément des PAS par la nature de sa conditionnalité, appropriée par les pays partenaires plutôt que définie depuis l'étranger (voir encadré 1). Corollairement, le processus d'adoption de cette conditionnalité fait l'objet d'une véritable négociation plutôt que d'une imposition. Comme mentionné précédemment, ce processus de négociation, communément appelé «dialogue politique » (policy dialogue), est souvent complété par des appuis d'assistance technique (partage des connaissances) ou de renforcement institutionnel afin de permettre aux pays partenaires de bénéficier des enseignements tirés ailleurs dans la mise en œuvre de réformes souvent complexes, en particulier dans le domaine des finances publiques. De par ces dimensions extrafinancières, l'aide budgétaire générale constitue donc bien davantage qu'un transfert de ressources financières ${ }^{1}$. Ces dimensions complémentaires sont brièvement décrites dans les deux prochaines sections, avant que ne soit abordée la question des résultats obtenus par l'aide budgétaire.

Pour une description plus détaillée de l'approche par le SECO de l'application de l'aide budgétaire générale, voir: SECO, SECO's Strategy for General Budget Support, Bern, SECO, n.d., disponible sur <http://www.seco-cooperation.admin.ch/shop/00008/00017>. 


\section{Encadré 1: La conditionnalité, un concept en évolution constante}

L'évolution de la pratique de la conditionnalité au cours de ces dix dernières années mériterait un traitement plus approfondi et ne peut être abordée que partiellement ici. II faut noter toutefois que tant la Banque mondiale que le Fonds monétaire international, cibles privilégiées des critiques des PAS, ont joué eux-mêmes un rôle pilote dans la réflexion sur la réforme de la conditionnalité. A la suite d'une évaluation critique de la conditionnalité telle que pratiquée jusque dans les années 1990 (rapport Assessing Aid: What Worls, What Doesn't, and Why, New York, Oxford University Press, 1998), la Banque mondiale a arrêté cinq principes directeurs devant guider la négociation de conditionnalités:

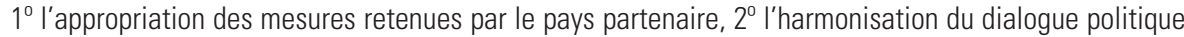
sur la conditionnalité autour d'un cadre commun (comme les Performance Assessment Frameworks PAFs - présents dans tous les pays où la Suisse intervient), $3^{\circ}$ I'adéquation des conditionnalités au contexte spécifique du pays, $4^{\circ}$ la limitation des déclencheurs de décaissements à un nombre limité de mesures prioritaires et $5^{\circ}$ la transparence des conditionnalités et la prévisibilité des décaissements liés aux examens de performance ${ }^{a}$. Ces principes sont largement reconnus par la plupart des bailleurs, y compris la Suisse. Le débat sur la conditionnalité n'est pas clos pour autant. Des divergences de vues persistent sur la nature de la conditionnalité. Pour certains, il faudrait se limiter à une conditionnalité purement politique et éviter toute volatilité dans les flux d'aide en déliant les décaissements de la performance. Ce n'est pas là le point de vue du SECO, pour lequel l'aspect performance est absolument essentiel, ne serait-ce que pour des raisons de responsabilités vis-à-vis des citoyens, tant dans les pays donateurs que dans les pays bénéficiaires. Un autre débat a trait au meilleur moyen de mesurer cette performance. La Commission européenne ambitionne d'évaluer autant que possible la performance à I'aune des OMD et pose comme conditions d'une partie de ses déboursements des indicateurs de résultats (accès aux services publics par exemple), ce qui est intéressant mais aussi exigeant en termes de fiabilité des systèmes statistiques du pays partenaire. Cette approche pose également des risques en matière de prévisibilité (difficulté de définir les cibles ex ante sans arbitraire) et d'attribution (notamment si les cibles sont manquées à cause de chocs exogènes). Une autre approche consiste à se limiter aux actions de politique économique et sociale pouvant être strictement attribuables à la responsabilité du gouvernement partenaire, ce dernier étant en fin de compte la partie contractuelle à un accord d'aide budgétaire. Le SECO a adopté de façon mesurée une approche mixte, avec l'application de tranches de performance basées sur des résultats mesurables mais aussi assez directement attribuables aux actions publiques ${ }^{b}$.

a Pour plus de détails sur l'application préliminaire de ces principes, voir: World Bank, Good Practice Principles for the Application of Conditionality: A Progress Report, Operations and Country Services, Washington, DC, The World Bank, November 2006.

b Sur ce sujet, voir différentes contributions dans: S. Koeberle, Z. Stavreski and J. Walliser (eds.), Budget Support as More Effective Aid? Recent Experiences and Emerging Lessons, Washington, DC, The World Bank, 2006.

\section{Soutenir un dialogue politique de qualité EST aussi une question d'organisation}

Très concrètement, dans les pays où la Suisse est active, le dialogue politique est tout d'abord organisé de façon thématique ou sectorielle, au sein de différents groupes de travail et commissions d'experts. Les participants incluent des techniciens des administrations publiques et des agences de développement, mais aussi des représentant de la société civile ou du secteur privé. Le travail des groupes ou commissions est ensuite synthétisé et discuté dans des examens annuels ou semestriels plus généraux, où sont aussi abordées des questions transversales, telles que la bonne gouvernance par exemple. Pour que ces processus en apparence complexes reposent sur des données fiables et puissent se concentrer sur les questions prioritaires, une bonne organisation est absolument essentielle. Le pays partenaire conduit le processus et est généralement secondé 
par un bailleur chef de file. La Suisse a eu le privilège d'assurer ce rôle dans plusieurs pays ces dernières années et elle s'est signalée comme un excellent facilitateur du dialogue politique.

\section{L'approche suisse du soutien aux réformes des finances publiques}

Le SECO place l'appui aux réformes de la gestion des finances publiques (communément abrégée selon l'acronyme anglais PFM pour public financial management) au centre de son programme de soutien macroéconomique. Son action dans ce domaine se déploie à plusieurs niveaux.

$\mathrm{Au}$ niveau international, le SECO participe régulièrement au forum du CAD consacré aux questions de PFM (Joint Venture on PFM). Avant tout, il a été une des agences fondatrices de l'initiative PEFA (Public Expenditure and Financial Accountability), qui a permis de développer non seulement un outil de diagnostic des finances publiques, mais aussi un cadre de référence pour le dialogue politique et la mobilisation de l'assistance technique dans le domaine $\mathrm{PFM}^{2}$. Forte de son expérience sur le plan conceptuel grâce à cet instrument, la Suisse a également promu son application dans un certain nombre de pays prioritaire, y compris ceux où elle n'applique pas l'aide budgétaire (Azerbaïdjan, Kirghizistan et Tadjikistan).

Au niveau des pays partenaires, le SECO attache une importance toute particulière aux réformes PFM dans le dialogue politique soutenu par l'aide budgétaire. Ainsi, les décaissements sont souvent liés à des éléments précis de la performance des pays partenaires en matière de réformes des finances publiques (par exemple taux d'exécution des dépenses prioritaires, mise en place de systèmes d'information visant la transparence budgétaire, renforcement des structures de contrôle budgétaire). En général, les spécialistes des bureaux de coopération suisses font partie des groupes thématiques suivant les questions de PFM et alimentent ainsi le dialogue politique y relatif.

Par ailleurs, le SECO accompagne pratiquement tout les appuis budgétaires suisses par des programmes de renforcement institutionnel ou d'assistance technique dans divers domaines liés à la gestion des finances publiques :

๖ programmation budgétaire (Nicaragua, Tanzanie et Mozambique);

๖ exécution budgétaire (Burkina Faso);

๖ évaluation de la dépense publique (Nicaragua et Tanzanie);

$\checkmark$ transparence et accès à l'information par des structures non gouvernementales (Burkina Faso);

- gestion de la dette publique (tous les pays d'intervention).

Toujours au niveau des pays partenaires, le SECO considère que les réformes des politiques et des administrations fiscales font partie intégrante d'un programme PFM. Ainsi, en complément à ses appuis budgétaires, des programmes d'assistance technique reçoivent le soutien de la Suisse en matière de réforme de l'administration fiscale, au Mozambique notamment (voir pp. 215-216).

2 Pour plus de renseignements sur l'initiative PEFA, voir: <http://www.pefa.org >. 
Outre les bénéfices directs attendus de l'aide budgétaire, les bailleurs membres du CAD ont également identifié l'aide programmatique comme une application directe de leurs engagements pour une coopération plus efficace, dans la mesure où l'instrument incorpore pratiquement tous les principes d'action identifiés à cette fin: une plus grande appropriation par les pays partenaires, un meilleur alignement des interventions extérieures sur les priorités définies localement, une plus grande harmonisation des pratiques des bailleurs, un accent plus marqué sur les résultats de la coopération ou encore la responsabilisation mutuelle des pays partenaires et des bailleurs face aux résultats de la coopération ${ }^{3}$.

Pour sa part, la Suisse a commencé à appliquer l'instrument de l'aide budgétaire dans sa forme actuelle à la fin des années 1990 au Mozambique. Mais son application demeure très sélective: elle concerne six pays partenaires ${ }^{4}$, pour un montant de décaissements annuels avoisinant les 40 millions de francs suisses, soit moins de $3 \%$ du volume total de l'aide publique bilatérale suisse au développement ${ }^{5}$. Ce pourcentage stagne depuis quelques années. D'autres pays du CAD, tels la Grande Bretagne, la Suède ou les Pays-Bas, accordent des volumes plus importants et croissants à l'aide budgétaire. Certains pays qui n'avaient jusqu'à récemment pas eu recours à cet instrument, comme l'Allemagne ou le Japon, ont commencé à le mettre en œuvre depuis quelques années. Enfin, une minorité de membres du CAD, dont avant tout les Etats-Unis, renoncent encore à l'instrument même si l'agence USAID soutient certains programmes d'aides budgétaires sectorielles. Malgré la proportion encore assez modeste d'APD bilatérale investie sous forme d'aide budgétaire générale, il faut noter que dans les pays où la Suisse applique l'aide budgétaire, cet instrument détient une part importante du volume du programme suisse (entre $30 \%$ et $50 \%$ ). Par ailleurs, l'OCDE a publié des bonnes pratiques en matière d'aide budgétaires dans le cadre de la mise en œuvre de la Déclaration de Paris ${ }^{6}$.

\section{Premier bilan intermédiaire}

\section{Un impact final encore difficilement attribuable...}

Quelques années après le début de la mise en œuvre de l'aide budgétaire, un groupe d'une vingtaine de pays donateurs et d'institutions multilatérales, dont la Suisse, se sont réunis sous l'égide du CAD pour commanditer une évaluation indépendante de l'instrument de l'aide budgétaire générale et mesurer si les

3 Ces principes ont été entérinés dans la Déclaration de Paris, signée en mars 2005 par un grand nombre de pays donateurs, dont la Suisse (voir <http://www.oecd.org/dac $>$ ). La déclaration prévoit notamment que pas moins de $66 \%$ du volume d'aide bilatérale devraient être alloués sous forme d'appuis programmes. Un premier bilan de la mise en œuvre de la déclaration sera établi lors d'une conférence internationale à Accra en janvier 2008.

4 Le Burkina Faso, le Ghana, le Mozambique, le Nicaragua, la Tanzanie et, plus récemment, le Bénin.

5 En 2006, la Suisse a fourni 41 millions de francs suisses d'aide budgétaire et a notifié un volume d'aide publique au développement se montant à 2 milliards (y compris les remises de dette).

6 Voir: OECD, Harmonizing Donor Practices for Effective Aid Delivery, vol. 2: Budget Support, DAC Guidelines and Reference Series, Paris, OECD, 2006. 
attentes placées dans cette nouvelle forme de coopération avaient pu être satisfaites. Une première dans son genre par l'ampleur du champ d'analyse et le nombre d'acteurs impliqués, cette évaluation a porté sur une étude approfondie des modalités de mise en œuvre de l'aide budgétaire générale dans sept pays partenaires jusqu'en $2004^{7}$.

Etant donné la complexité de la tâche et la multiplicité des questions à examiner, les évaluateurs ont défini un cadre d'analyse à plusieurs niveaux (voir schéma 1), qui sont repris successivement ci-dessous. En commençant - en aval de la chaîne de causalité - par la question principale, à savoir celle de l'impact sur la croissance et la réduction de la pauvreté, il faut noter que cette évaluation rejoint la conclusion de nombreuses études sur l'impact final de l'aide au développement. Les résultats observés en matière de croissance accélérée ou de réduction de la pauvreté (données à plus basses fréquences, et donc moins disponibles) ne sont pas directement attribuables à l'aide budgétaire, principalement à cause d'écueils méthodologiques difficilement contournables. L'impossibilité de dériver une corrélation significative entre volume d'aide (dont l'aide budgétaire) et croissance économique a alimenté de nombreuses argumentations critiques envers la coopération au développement ${ }^{8}$. Les raccourcis sont parfois malheureux puisque l'absence de corrélation statistiquement démontrable dans une étude transversale sur de nombreux pays et de longues périodes ne signifie en tout cas pas qu'il y ait un effet négatif de l'aide sur le développement économique, ni même que l'aide n'ait pas d'effet positif dans un pays donné, cette information étant perdue à cause des effets d'agrégation dans le temps et dans l'espace.

Ainsi, même si dans la plupart des pays examinés par l'évaluation de l'aide budgétaire on note une accélération de la croissance, sur le plan empirique celle-ci s'avère difficilement attribuable à l'aide ${ }^{9}$. Dans les pays où la Suisse applique l'aide budgétaire, on a pu noter une augmentation de la croissance et, dans les cas où la pauvreté était mesurée, une baisse, bien qu'encore modérée, de cette dernière. Il serait trop rapide d'inférer pour autant un effet de causalité directe sans examens plus approfondis. Au Mozambique par exemple, la Direction d'analyse économique du Ministère de l'économie a procédé à une étude quantitative décomposant la forte croissance des dix dernières années, et a conclu à un impact positif de l'aide, parmi d'autres variables ${ }^{10}$.

7 Le Burkina Faso, le Malawi, le Mozambique, le Nicaragua, le Rwanda, l'Ouganda et le Vietnam. Le document de synthèse de l'évaluation (Evaluation of General Budget Support: Synthesis Report) et les études préparatoires effectuées par un consortium d'experts dirigé par l'International Development Department (IDD) de l'Université de Birmingham sont disponibles sur <http://www.oecd.org/ document/51/0,3343,en_21571361_34047972_36556979_1_1_1_1,00.html>. Un bilan qualitatif de l'aide budgétaire avait été dressé dans: S. Koeberle, Z. Stavreski and J. Walliser (eds.), op. cit.

8 Voir par exemple l'ouvrage suivant, dont certaines conclusions ont récemment trouvé un large écho dans la presse suisse: W. Easterly, The White Man's Burden, New York, The Penguin Press, 2006.

9 Voir: M. Clemens, S. Radelet and R. Bhavnani, Counting Chickens When They Hatch: The Shortterm Effect of Aid on Growth, Working Paper, $\mathrm{n}^{\circ}$ 44, Washington, DC, Center for Global Development, 2004, disponible sur <http://www.cgdev.org>. Les auteurs utilisent une méthodologie économétrique relativement sophistiquée pour étudier l'impact de différentes modalités d'aide sur la croissance. Ils démontrent que certains types d'aide (dont l'aide budgétaire) peuvent avoir un impact positif sur la croissance à court terme. Ils trouvent toutefois qu'à partir d'un certain niveau, les flux d'aide produisent des rendements décroissants.

10 C. Arndt, S. Jones and F. Tarp, Aid and Development: The Mozambican Case, Discussion Papers, $\mathrm{n}^{\mathrm{o}}$ 27E, National Directorate of Studies and Policy Analysis, Ministry of Development, Republic of Mozambique, May 2006, disponible sur <http://www.mpd.gov.mz/gest/English.htm> > publications. 
En poursuivant l'analyse le long du chemin de la causalité, il faut dès lors examiner de plus près les cas particuliers de chaque pays ainsi que des variables intermédiaires au travers desquelles on peut raisonnablement déduire que l'aide, et l'aide budgétaire en particulier, peut avoir un effet sur des variables d'impact telles que la croissance ou le niveau de pauvreté.

\section{Schéma 1: cadre d'analyse pour mesurer les effets de l'aide budgétaire générale}

0. Conditions préalables

1. Inputs

2. Effets immédiats

3. Effets à court terme

4. Résultats à moyen terme

5. Impact
- Performance et politique macroéconomiques

- Niveau de gouvernance

- Niveau de gestion des finances publiques

- Engagement démontré pour une politique de croissance et de réduction de la pauvreté

- Ressources financières

- Dialogue politique et conditionnalité

- Assistance technique et renforcement institutionnel

- Accroissement de la part des financements extérieurs passant par le budget national

- Meilleure prévisibilité du financement extérieur

- Concentration du dialogue de politique et de la conditionnalité sur des thèmes prioritaires

- Meilleure coordination des bailleurs et alignement sur les priorités définies dans les pays partenaires

- Renforcement de la discipline budgétaire

- Accroissement de la part des secteurs prioritaires dans les allocations budgétaires

- Renforcement des systèmes de gestion des finances publiques

- Plus grande responsabilisation des Etats dans l'exécution de leur budget (obligation de rendre compte)

- Meilleure cohérence entre les objectifs de politiques sectorielles et le cadre macroéconomique

- Stabilité macroéconomique

- Conditions-cadres pour l'investissement privé

- Efficacité des services publics

- Croissance économique

- Réduction de la pauvreté

Source: DAC Network on Development Evaluation, Evaluation of General Budget Support, <http://www.oecd.org/document/ 51/0,3343,en_21571361_34047972_36556979_1_1_1_1,00.html>. 


\section{... qui contraste avec des résultats intermédiaires probants}

En ce qui concerne l'effet sur la stabilité macroéconomique et financière, une condition-cadre indispensable à la croissance, l'analyse démontre que l'aide budgétaire joue un rôle positif en raison de l'importance centrale que cette question occupe dans le dialogue politique. L'objectif n'est plus seulement, comme au temps de l'aide à la balance des paiements, de stabiliser l'économie, mais aussi d'assurer la pérennité de cette stabilité malgré des défis structurels et sociaux souvent importants. Ainsi, au travers du dialogue politique facilité par le processus d'aide budgétaire, la cohérence entre les priorités sectorielles (par exemple le recrutement d'enseignants afin d'augmenter les taux de scolarisation) et le maintien des équilibres macroéconomiques (dans ce cas, une gestion maîtrisée de l'augmentation de la masse salariale) est constamment mise à l'épreuve et discutée. D’une manière générale, dans les pays où la Suisse est présente avec l'aide budgétaire, on n'a pas constaté de dérapage au niveau des dépenses publiques ou du solde budgétaire, à l'image du Mozambique (voir graphique 1).

\section{Graphique 1: Mozambique - évolution des agrégats budgétaires, 1999-2006}

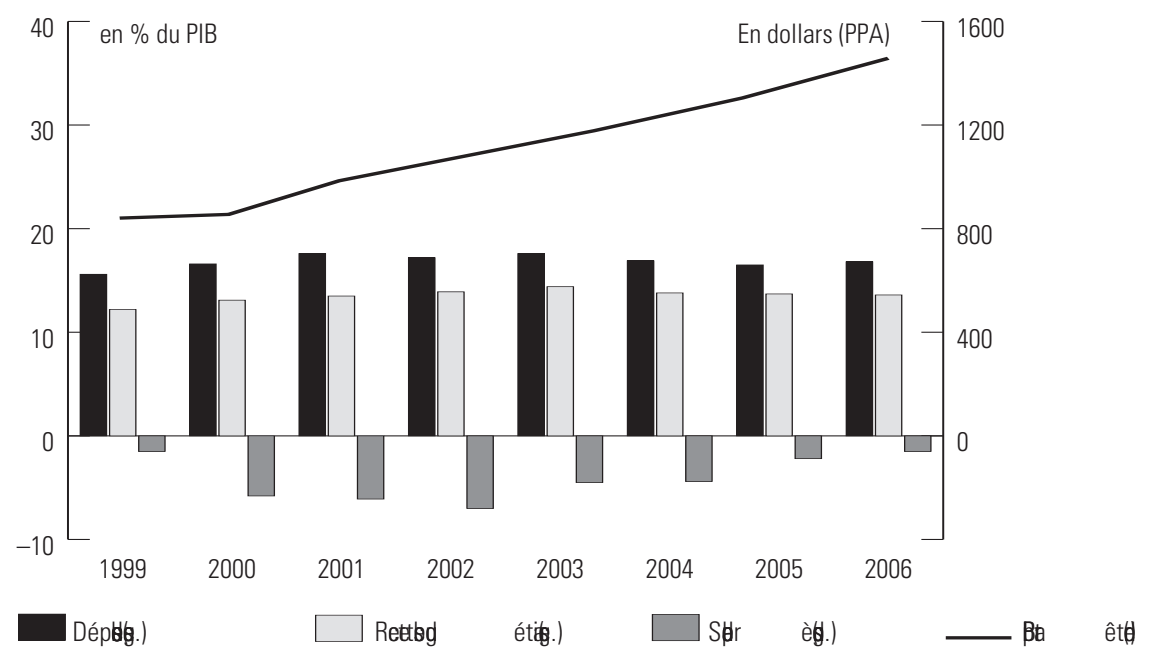

Source: Graphique élaboré par J.-L. Bernasconi à partir de données du FMI.

Légende: Ce graphique montre plusieurs évolutions entre 1999 et 2006, période où la part de l'aide budgétaire a augmenté relativement à l'aide totale: $1^{\circ}$ le PIB par tête en valeur réelle s'est accru, de $3 \%$ à $4 \%$ par an, $2^{\circ}$ les dépenses publiques hors projets financés de l'extérieur (colonnes noires) ont augmenté mais restent soutenables, $3^{\circ}$ bien qu'encore insuffisant, l'effort de mobilisation des recettes domestiques n'a pas diminué malgré les apports extérieurs, $4^{\circ}$ grâce aux appuis budgétaires sous forme de dons, le besoin de financement de l'Etat a progressivement diminué.

En ce qui concerne les conditions-cadres pour le développement de l'investissement, en particulier du secteur privé, l'aide budgétaire joue globalement un rôle favorable car elle permet de concentrer le dialogue politique sur un nombre limité de réformes prioritaires. Les premiers résultats sont là pour le prouver puisqu'on note, dans l'enquête annuelle de la Banque mondiale relative aux contraintes pesant sur la marche des affaires, des progrès notoires dans certains pays africains où l'aide budgétaire a pris une ampleur croissante ces dernières 
années (ainsi, par exemple, le Ghana et la Tanzanie figurent parmi les dix pays ayant fait le plus de progrès en la matière en 2006) ${ }^{11}$.

Un effet plus directement mesurable de l'aide budgétaire générale est celui qu'elle peut exercer sur la composition des dépenses budgétaires et la gestion des finances dans les Etats partenaires. La part relative des dépenses prioritaires dans les budgets nationaux a augmenté régulièrement dans les pays bénéficiant de l'aide budgétaire. Ainsi, l'efficacité allocative (c'est-à-dire l'adéquation entre allocations budgétaires et domaines prioritaires) des politiques de développement s'est régulièrement accrue. Certes, à cause des inerties que l'on ne peut que progressivement renverser, les réallocations budgétaires sont graduelles. Au Mozambique par exemple, les dépenses prioritaires (santé, éducation, infrastructure de base, gouvernance et justice) ont passé de $61 \%$ à $70 \%$ du budget entre 2000 et 2006. Les budgets des pays concernés sont souvent très réduits et certaines dépenses difficilement compressibles à court terme constituent une portion importante de la dépense publique totale. Mais la perception, parfois véhiculée par les critiques de l'aide budgétaire, selon laquelle ces ressources alimenteraient les dépenses non productives des Etats est infirmée par l'évaluation de l'IDD et par d'autres analyses. Ainsi, dans les six pays où la Suisse met en œuvre l'aide budgétaire, les dépenses militaires par exemple se situent en moyenne à $1,1 \%$ du produit intérieur brut (PIB) contre des valeurs oscillant entre 2,5 et $3 \%$ pour la moyenne des pays à faible revenu, voire $3 \%$ pour certains pays à revenu moyen ou membres de l'OCDE. Cette proportion a en outre diminué de $3 \%$ par an depuis 2000 dans ces pays d'intervention ${ }^{12}$.

L'efficacité opérationnelle dans la gestion des ressources budgétaires s'est également améliorée car le dialogue politique porte une attention croissante à l'efficacité, à la transparence et à l'intégrité de la gestion financière de l'Etat. Certes, l'exigence accrue des bailleurs envers les Etats partenaires pour que ceux-ci rendent des comptes quant à l'exécution de leur budget pourrait aussi comporter des risques, dans la mesure où elle évincerait la responsabilisation vis-à-vis des institutions et des parties prenantes locales (par exemple les parlements nationaux). Certaines expériences montrent toutefois que ces processus peuvent se renforcer mutuellement, par exemple lorsque les bailleurs se réfèrent aux examens des institutions nationales de vérification des comptes, telles les cours des comptes ou les instituts d'audit public. Le renforcement et l'indépendance de ces institutions font d'ailleurs le plus souvent partie intégrante du dialogue politique promu par l'aide budgétaire. L'examen régulier de la gestion financière tout au long du cycle budgétaire est d'une importance absolument cruciale si l'on entend que l'aide budgétaire amène les bénéfices attendus (voir encadré 2). Les réformes de la gestion des finances publiques doivent ainsi se trouver impérativement au centre du dialogue politique et recevoir une attention particulière dans les programmes de renforcement institutionnel et d'assistance technique. C'est en tout cas l'approche adoptée par la Suisse, telle que décrite précédemment.

11 Banque mondiale, Doing Business. Pratique des affaires en 2006, Paris, Eska, 2006.

12 Calculs du SECO, selon les données compilées par l'Institut international de recherche sur la paix de Stockholm, disponibles sur $<$ http://www.sipri.org $>$. 


\section{Encadré 2: La nécessité d'une approche intégrée de la gestion des finances publiques: l'exemple du Bénin}

Au milieu des années 1990, le Bénin est rapidement apparu comme un candidat privilégié à l'aide programmatique. La performance économique du pays était généralement bonne et la gouvernance politique reposait sur des bases démocratiques reconnues par les bailleurs. A juste titre, les efforts du gouvernement et des bailleurs se sont ainsi concentrés sur l'amélioration de la gestion budgétaire afin que les appuis programmatiques déploient leurs meilleurs effets. Ils ont insisté avant tout sur la programmation budgétaire, choix très logique puisque l'on se trouvait alors au moment de l'émergence des stratégies de réduction de la pauvreté (SRP) et du lancement de I'Initiative de désendettement en faveur des pays pauvres très endettés (PPTE). Tant les stratégies que l'initiative mettaient un accent alors novateur sur une plus grande adéquation entre les allocations budgétaires des Etats et les objectifs de développement (en particuliers les Objectifs du Millénaire pour le développement, OMD). C'est ainsi que fut lancée au Bénin l'approche "budget-programme», dans laquelle les arbitrages budgétaires annuels classiques sont redéfinis en programmes à moyen terme, avec des indicateurs de résultats précis liés aux ressources mises à disposition par le budget national dans un secteur donné. Dans le cas de l'éducation par exemple, au sein du programme "éducation primaire», on met en relation les "intrants" (salles de classe, postes d'enseignement, formation professionnelle, matériel scolaire, etc.) avec les résultats attendus (par exemple taux bruts de scolarisation, taux de redoublement, etc.), eux-mêmes désagrégés par genre. Les responsables du ministère en question sont ainsi à même de simuler l'effet d'une variation des crédits alloués. Au cours de la dernière décennie, pratiquement tous les ministères béninois ont adopté l'approche budget-programme, le pays faisant ainsi œuvre de pionnier dans la sous-région en matière de programmation de la dépense publique.

Malheureusement, les faiblesses dans d'autres aspects de la gestion budgétaire n'ont permis aux budgets-programmes de jouer leur rôle qu'imparfaitement jusqu'à peu. Ainsi, une surévaluation chronique des recettes a compliqué la gestion de l'exécution du budget et de la trésorerie. Les crédits étant rationnés, des pratiques se sont développées selon lesquelles les dépenses étaient engagées selon des procédures en principe extraordinaires et les allocations budgétaires prévues dans la loi de finances perdaient peu à peu de leur signification, les budgets-programmes courant dès lors le risque de n'être que des exercices de style. Heureusement, les nouvelles autorités béninoises en place depuis 2006 ont reconnu ce problème et pris les mesures correctrices qui s'imposent. Cet exemple montre toutefois l'importance d'appréhender les forces et faiblesses de la gestion budgétaire sur l'ensemble de la «chaîne de production".

En ce qui concerne l'effet de l'aide budgétaire sur les services publics prioritaires (avant tout les services de santé et d'éducation), on note une augmentation nette de leur volume. Par contre, l'effet sur leur qualité est ambigu. Cela s'explique par une logique de séquencement. Les Objectifs du Millénaire pour le développement (OMD) ont en premier lieu stimulé un effort porté sur l'amélioration quantitative de certains indicateurs (par exemple les taux bruts de scolarisation). L'action publique s'est ainsi d'abord concentrée sur la mobilisation de ressources permettant d'accompagner cette croissance quantitative (recrutement d'enseignants, construction de salles de classe). Par contre, cet effort ne pourra être soutenable que s'il est rapidement accompagné par des mesures visant à assurer que la qualité des services suive le rythme de leur expansion (pour reprendre l'exemple de l'éducation, on peut penser ici à la formation des enseignants ou à la mise en place de débouchés suffisants au sein du système d'enseignement postprimaire).

Finalement, l'évaluation a permis également de réexaminer la relation entre aide budgétaire générale et aide sous forme de projets. On a trop rapidement 
voulu présenter ces deux modalités comme substitutives alors qu'elles devraient être complémentaires. L'amélioration indiscutable de la coordination entre les bailleurs produite par l'aide budgétaire a à son tour eu des retombées positives sur la manière dont est gérée la coopération plus traditionnelle sous forme de projets. Qu'ils fournissent de l'aide budgétaire ou non, les bailleurs conservent une majorité de leur portefeuille d'intervention sous forme de projets cloisonnés. Toutefois, une prise de conscience s'opère graduellement à propos des inefficacités liées aux structures de gestion parallèles, surtout dans des pays où les capacités sont encore très limitées. De même, les bailleurs ont pris conscience de l'importance, en matière de transparence et de crédibilité de leur action, d'une intégration des interventions dans les budgets nationaux, même si ces ressources sont affectées à des projets bien précis. Au-delà de ces questions d'harmonisation, les synergies potentielles entre projets et aide budgétaire restent encore trop peu exploitées. Le projet est sans doute la modalité la plus adaptée pour tenter des approches innovatrices à petite échelle. Il s'impose généralement aussi si les partenaires sont des intervenants du secteur privé ou de la société civile. Par contre, si ces innovations font leurs preuves et méritent d'être amplifiées, l'aide budgétaire est alors une caisse de résonance idéale pour diffuser les expériences positives du terrain et favoriser leur application à plus grande échelle. C'est en tout cas l'approche de la Suisse. A titre d'exemple, on peut citer ce type de synergie dans le domaine de la santé en Tanzanie. Au niveau des projets, la Suisse a soutenu des expériences novatrices relatives à la fabrication et à la distribution de moustiquaires imprégnées. Pour pérenniser cette action, il était impératif que la politique sanitaire promeuve cette approche par la sensibilisation des utilisateurs potentiels dans les centres de santé, une politique de tarification des moustiquaires, etc. Pour ce faire, la Suisse a fourni un appui budgétaire sectoriel et a pu ainsi se voir attribuer le rôle de chef de file au sein de la commission thématique relative à la politique de santé. Finalement, l'approche ne peut être multipliée à l'échelle nationale que si les allocations budgétaires au système de santé augmentent et les systèmes de gestion des finances publiques s'améliorent. C'est à ce stade qu'intervient l'aide budgétaire générale. Le résultat est probant: la mortalité infantile a diminué en Tanzanie, ce que nombre d'experts attribuent à une meilleure mise en œuvre de la politique sanitaire, notamment en matière de lutte contre le paludisme.

\section{Les défis restants: gérer les risques et développer les potentialités}

L'évaluation menée sous l'égide du CAD a confirmé que l'aide budgétaire est un instrument qui comporte des risques. Mais, contrairement à l'image de la «boîte noire» qu'on associe parfois à l'aide budgétaire, ces risques ne sont ni diffus ni insurmontables. Ils peuvent être clairement identifiés au préalable et des stratégies de mitigation peuvent être adoptées pour limiter leur impact potentiel.

En premier lieu, le risque de corruption vient immédiatement à l'esprit quant on songe à des ressources mises à la disposition de pays en voie de développement de façon non affectée pour être ensuite utilisées suivant des procédures nationales. L'évaluation par l'IDD réalisée dans le cadre de l'OCDE a eu le grand mérite de démystifier cette question. Tout d'abord, il s'est avéré que l'aide bud- 
gétaire n'est pas plus vulnérable au risque de corruption que l'aide par projets, surtout lorsque cette dernière consiste en de grands projets d'infrastructure notamment. En outre, il est possible de bien identifier les composantes du risque dit «fiduciaire» et de prendre des mesures pour le limiter en incluant ses dimensions les plus importantes dans le dialogue politique et les conditionnalités de l'aide budgétaire.

L'aide budgétaire est souvent perçue comme un instrument particulièrement vulnérable aux risques de mauvaise gouvernance économique et politique, la «traçabilité» des fonds étant a priori moins facile qu'elle ne l'est pour le financement de projets cloisonnés. En fait, les risques de mauvaise gouvernance économique liés à l'aide budgétaire s'appliquent à toutes les ressources alimentant le budget (revenus fiscaux, etc.). C'est donc dans les faiblesses des systèmes de gestion des finances publiques que résident ces risques, dits «fiduciaires». L'appréciation des risques fiduciaires courus par l'ensemble des ressources budgétaires se justifie d'autant plus que les «victimes» potentielles ne sont pas seulement les contribuables des pays donateurs, mais avant tout les citoyens des pays partenaires. Le risque fiduciaire lié à l'aide budgétaire comporte quatre dimensions:

1. Risque de transfert: ce risque est proprement lié au transfert de fonds de l'aide budgétaire. Il décrit la possibilité que ces fonds soient détournés avant d'atteindre les comptes du trésor. Des audits extérieurs sont entrepris par les bailleurs pour contrôler ce risque, qui ne s'est jamais concrétisé dans les pays d'intervention du SECO.

2. Risque d'exécution non planifiée: ce risque décrit la possibilité que le budget ne soit pas exécuté conformément au budget voté par le parlement.

3. Risque de non-enregistrement: ce risque inclut une comptabilisation inadéquate des flux financiers, ou encore le fait que les vérifications (contrôles internes, audit externes) soient inexistantes ou ne répondent pas à des exigences de qualité et de délais nécessaires.

4. Risque d'utilisation sous-optimale des ressources («value for money»): cette notion recouvre le concept de value for money, selon lequel les ressources doivent être engagées selon les principes d'économie (achats au moindre coût), d'efficience (services publics effectivement fournis) et d'efficacité (par rapport aux objectifs de développement).

L'appréciation et la limitation du risque reposent sur trois types d'outils: $1^{\circ}$ le diagnostic a priori, $2^{\circ}$ le contrôle simultané, et $3^{\circ}$ les audits et évaluations a posteriori. Le diagnostic a priori est particulièrement important en ce qui concerne les appuis budgétaires du SECO. En effet, le SECO considère avec attention les conditions préalables et n'entre en matière pour des appuis budgétaires que dans des environnements où le niveau de risque fiduciaire est acceptable, où les autorités du pays partenaire ont un programme crédible de renforcement des finances publiques, agréé avec les institutions financières multilatérales, et où le risque fiduciaire est régulièrement évalué et les progrès réalisés pour le limiter peuvent être observés.

A cette fin, des diagnostics externes et indépendants sont en général mandatés par les bailleurs en consultation avec les autorités et entrepris de façon indépendante. Ils suivent la méthodologie PEFA, développée avec le soutien de la 
Suisse, et permettent de mesurer le risque à l'entrée ainsi que le progrès des mesures de renforcement des finances publiques. Par ailleurs, dans les pays où le SECO est présent, les systèmes nationaux de gestion et de contrôle des finances publiques comportent des contrôles simultanés de la dépense qui limitent le risque. Il est évident que la qualité des systèmes nationaux de contrôle reflète aussi les faiblesses des capacités administratives en présence dans la plupart des pays partenaires. Ces capacités sont toutefois évaluées régulièrement et le fonctionnement effectif des organes de contrôle est au cœur du dialogue politique entre bailleurs et autorités.

Cela étant, il ne serait pas raisonnable d'attendre de l'aide budgétaire qu'elle permette de résoudre toutes les questions de corruption. Les autres dimensions de la corruption (dessous-de-table, «petite corruption», pots-de-vin, caisses noires, trafics d'influence, etc.), quoique très dommageables au développement des pays où elles se manifestent, ne posent pas un risque plus direct à l'aide budgétaire qu'à d'autres types de coopération. Le dialogue politique auquel l'aide budgétaire donne lieu peut, par contre, permettre de promouvoir un débat transparent sur ces autres dimensions de la corruption. Au Nicaragua ou au Burkina Faso par exemple, des réformes institutionnelles (telle la réforme du système judiciaire ou de la fonction publique) ont été retenues comme conditionnalités de l'aide budgétaire. L'impact réel de telles mesures sur le niveau de corruption devra être observé régulièrement et l'aide budgétaire ne saurait être considérée comme la panacée contre la corruption. Elle contribue en revanche à l'amélioration du climat anticorruption par l'effet direct qu'elle exerce sur la gestion des finances publiques.

Un second type de risque a trait à des vulnérabilités macroéconomiques. D'une part, un afflux massif de financement extérieur sous forme d'aide (en général, y inclus l'aide budgétaire) pourrait déclencher une appréciation réelle de la monnaie locale et donc une perte de compétitivité causée par l'augmentation soudaine de la demande pour des biens et des services des secteurs protégés (ou non échangeables). Ce type de phénomène n'a pas encore été empiriquement démontré pour des pays ayant reçu des flux importants d'aide. Le risque ne peut être écarté pour autant et renforce l'importance qu'il faut accorder à la gestion macroéconomique dans le cadre du dialogue politique. Un risque associé est celui de l'éviction de l'investissement privé. Afin précisément d'éviter la perte de compétitivité liée au «syndrome hollandais ${ }^{13}$, les autorités monétaires du pays bénéficiaire peuvent être amenées à «stériliser» l'impact par une politique monétaire excessivement restrictive, qui entraînerait une augmentation des taux d'intérêt et ainsi un découragement du crédit à l'économie privée. Au Mozambique, par exemple, on a pu observer que la banque nationale a effectivement stérilisé les afflux d'aide à court terme, agissant ainsi sur le niveau des taux d'intérêt qui demeurent élevés. Toutefois, il n'est pas correct d'attribuer cette

13 Certains auteurs parlent de «syndrome hollandais » lié à l'aide dans la mesure où l'impact macroéconomique de l'afflux de ressources s'apparente à celui généré par la découverte et l'exploitation d'une ressource naturelle. (Rappelons que l'expression «syndrome hollandais», apparue dans les années 1960 lorsque les richesses des Pays-Bas augmentèrent à la suite de la découverte de grands gisements de gaz dans le pays et en mer du Nord, désigne par extension les conséquences nuisibles provoquées par une augmentation significative des recettes d'un pays.) Voir par exemple: A. Prati and T. Tressel, Aid Volatility and Dutch Disease: Is There a Role for Macroeconomic Policies?, IMF Working Paper, doc. 06/145, Washington, DC, International Monetary Fund, 2006. 
évolution à l'aide seule, le pays ayant été soumis à une série de chocs exogènes de nature inflationniste (prix de l'énergie, sécheresse) qui nécessitaient une politique monétaire plutôt prudente.

Un autre risque relatif à la gestion macroéconomique a trait à la possibilité du problème d'aléa moral que susciterait la mise à disposition des ressources extérieures pour le budget par rapport à l'effort de mobilisation de ressources internes par le système fiscal national. Là également, ce risque ne s'est jusqu'ici pas vérifié, même si les taux de pression fiscale n'augmentent que très lentement dans les pays concernés (voir graphique 1). Le SECO s'engage dans tous les pays où il intervient pour que la question de la performance en matière de fiscalité soit pleinement intégrée dans le dialogue politique et que les besoins d'assistance technique soient satisfaits (voir pp. 215-216 pour l'exemple du Mozambique).

Une quatrième question souvent débattue dans le cadre des effets de l'aide budgétaire a trait à la décentralisation. En vertu du principe de subsidiarité, beaucoup de bailleurs gouvernementaux et non gouvernementaux ont placé la décentralisation au centre de leur stratégie de coopération. C'est également le cas de la Suisse dans la plupart des pays prioritaires d'intervention des programmes conjoints de la Direction du développement et de la coopération (DDC) et du SECO. L'aide budgétaire pourrait alors se révéler problématique dans la mesure où son action renforcerait les structures centralisatrices au détriment de la décentralisation ou déconcentration effective des services publics. Ce risque n'est pas à négliger mais ici également il peut être transformé en potentialité si l'on considère que l'aide budgétaire offre une plate-forme plutôt unique où des problèmes liés à la mise en œuvre de la décentralisation peuvent être abordés de front avec les autorités des pays partenaires au plus haut niveau. Ainsi, force est de constater que dans beaucoup de cas, la décentralisation promue par les bailleurs s'est concentrée de façon primordiale sur des questions politiques (promotion de la démocratie locale) ou administratives. Ces dimensions sont évidemment au cour de tout processus de décentralisation, mais si elles ne sont pas accompagnées, voire précédées, par une réflexion approfondie sur la décentralisation des processus de gestion budgétaire, les réformes seront inefficaces, voire contre-productives. Par l'accent qui est mis sur la question de la réforme des finances publiques, l'aide budgétaire peut contribuer à couvrir cet aspect important. Dans plusieurs cas, les questions de répartition de la fiscalité ou de transfert de compétences ont ainsi été incluses dans le dialogue politique associé à l'aide budgétaire.

L'aide budgétaire n'est pas non plus à l'abri des critiques adressées plus généralement aux programmes de coopération par rapport à des faiblesses dans l'obligation de rendre compte et les mécanismes incitatifs auxquels les acteurs sont soumis ${ }^{14}$. Ce risque se réfère au problème microéconomique bien connu de l'«agent-principal», où la relation entre un donneur d'ordre (principal) et

\footnotetext{
Voir: W. Easterly, op. cit., qui réinterprète à sa manière les approches institutionnalistes telles que celle de B. Martens, U. Mummert, P. Murrell et P. Seabright dans The Institutional Economics of Foreign Aid (Cambridge, Cambridge University Press, 2002) ou celle de J. Svensson dans «Absorption Capacity and Disbursement Constraints» (dans Financing Development: What Are the Challenges in Expanding Aid Flows?, Proceedings of the 3rd AFD-EUDN Conference, Paris, Agence française de développement, 2006).
} 
l'exécutant (agent) est teintée d'asymétrie dans l'information disponible, ce qui permet à l'agent de ne remplir qu'imparfaitement sa partie du contrat sans encourir de sanctions. Ce problème est en théorie particulièrement aigu pour les projets de coopération, étant donné la distance et le nombre d'intermédiaires qui séparent le principal (contribuable du pays bailleur) des bénéficiaires finaux. Le risque est encore amplifié par des problèmes d'action collective où de multiples agents de développement agissent de façon non cordonnée. Des mécanismes renforcés en matière de suivi, d'évaluation et d'obligation de rendre compte des résultats doivent être mis en place pour mieux orienter les incitations qui motivent l'action des agents impliqué dans les projets ou programmes. L'aide budgétaire implique par ailleurs des mécanismes de coordination entre bailleurs qui visent à réduire les inefficacités inhérentes à l'action collective.

Finalement, un dernier risque très important a trait au processus de dialogue politique lui-même, qui pourrait se résumer à des échanges de vues entre gouvernements bénéficiaires et bailleurs, laissant sur la touche les institutions démocratiques locales (parlements), les collectivités locales ou encore les représentants de la société civile ou du secteur privé. La participation des acteurs de la société civile pose un double défi: d'une part, favoriser l'accès à l'information sur le budget de l'Etat et les politiques macroéconomiques, d'autre part, renforcer les capacités des associations pour qu'elles puissent traiter cette information et l'utiliser pour leurs prises de position. La Suisse s'engage très concrètement dans tous les pays où elle applique l'aide budgétaire pour promouvoir la participation des acteurs non gouvernementaux au dialogue politique. En fin de compte, ceux-ci restent les mieux placés pour juger de l'efficacité de l'action publique dans leur pays et, ce faisant, de l'impact de l'aide budgétaire.

\section{Rôle et avantage pour la Suisse}

En dépit des difficultés de tirer une conclusion définitive sur l'impact de l'aide budgétaire, la conclusion globalement positive de l'évaluation menée sous l'égide du CAD quant aux résultats intermédiaires, ainsi que les expériences de terrain de la Suisse dans les pays où elle applique cet instrument, confirment que ce type de coopération se révèle essentiel dans des contextes précis où sont réunies certaines conditions préalables. Cela étant, même si l'instrument fait ses preuves, il faut continuellement examiner sa signification dans le cadre de l'aide bilatérale suisse. Premièrement, il s'agit d'évaluer quelle valeur ajoutée un bailleur, avec des volumes de financements parfois relativement modestes, peut apporter au processus. A cet égard, la Suisse a récemment assumé la présidence des groupes de bailleurs dans pas moins de quatre des six pays où l'instrument est mis en œuvre ${ }^{15}$. Elle est également cheffe de file d'importantes commissions thématiques ou sectorielles, où des questions de priorités des politiques de développement sont débattues. Cette expérience a prouvé qu'une présence forte sur le terrain au travers des bureaux de coopération assure une crédibilité reconnue par les partenaires locaux comme par les autres bailleurs. D'autre part, à cause des complémentarités et des synergies entre aide budgétaire et aide par projets mentionnées plus haut, la participation de la Suisse à ces processus augmente

15 Le Burkina Faso, le Mozambique, le Nicaragua et la Tanzanie. 
l'efficacité de l'ensemble du portefeuille de programmes de la coopération bilatérale. L'aide budgétaire est ainsi devenue un pilier central de la coopération dans les pays où elle est appliquée. Le défi reste dès lors d'en améliorer encore l'efficacité pour éviter un renversement du paradoxe «micro-macro» mentionné au début de cet article, à savoir une situation où les pays afficheraient de bonnes performances au niveau agrégé alors même que ces performances ne trouveraient pas de traduction tangible sur le terrain. La Suisse ne peut ni ne doit relever ce défi seule mais, grâce à sa forte présence et à son expérience de terrain, elle peut continuer à contribuer substantiellement à de meilleurs résultats.

\section{Bibliographie sélective}

Clemens, M., S. Radelet and R. Bhavnani, Counting Chickens When They Hatch: The Short-term Effect of Aid on Growth, Working Paper, $\mathrm{n}^{\circ}$ 44, Washington, DC, Center for Global Development, 2004, disponible sur <http://www.cgdev.org $>$.

Easterly, W., The White Man's Burden, New York, The Penguin Press, 2006.

International Development Department (IDD) and Associates, Evaluation of General Budget Support: Synthesis Report, Birmingham, University of Birmingham, May 2006.

Koeberle, S., Z. Stavreski and J. Walliser J. (eds.), Budget Support as More Effective Aid? Recent Experiences and Emerging Lessons, Washington, DC, The World Bank, 2006.

Martens, B., U. Mummert, P. Murrell and P. Seabright, The Institutional Economics of Foreign Aid, Cambridge, Cambridge University Press, 2002.

OECD, Harmonizing Donor Practices for Effective Aid Delivery, vol. 2: Budget Support, DAC Guidelines and Reference Series, Paris, OECD, 2006.

Prati, A. and T. Tressel, Aid Volatility and Dutch Disease: Is There a Role for Macroeconomic Policies?, IMF Working Paper, doc. 06/145, Washington, DC, International Monetary Fund, 2006.

Svensson, J., «Absorption Capacity and Disbursement Constraints», Financing Development: What Are the Challenges in Expanding Aid Flows?, Proceedings of the 3rd AFD-EUDN Conference, Paris, Agence française de développement, 2006.

World Bank, Good Practice Principles for the Application of Conditionality: A Progress Report, Operations and Country Services, Washington, DC, The World Bank, November 2006. 